\title{
Essential Role of Phox2b-Expressing Ventrolateral Brainstem Neurons in the Chemosensory Control of Inspiration and Expiration
}

\author{
Nephtali Marina, ${ }^{1 *}$ Ana P. Abdala, ${ }^{2 *}$ Stefan Trapp, ${ }^{3}$ Aihua Li, ${ }^{4}$ Eugene E. Nattie, ${ }^{4}$ James Hewinson, ${ }^{2}$ Jeffrey C. Smith, ${ }^{5}$ \\ Julian F. R. Paton, ${ }^{2}$ and Alexander V. Gourine ${ }^{1}$ \\ ${ }^{1}$ Neuroscience, Physiology and Pharmacology, University College London, London WC1E 6BT, ${ }^{2}$ Department of Physiology and Pharmacology, Bristol Heart \\ Institute, University of Bristol, Bristol BS8 1TD, ${ }^{3}$ Biophysics Section, Department of Surgery and Cancer, Imperial College London, South Kensington \\ Campus, London SW7 2AZ, United Kingdom, ${ }^{4}$ Department of Physiology, Dartmouth Medical School, Lebanon, New Hampshire 03756-0001, and ${ }^{5}$ Cellular \\ and Systems Neurobiology Section, National Institute of Neurological Disorders and Stroke, National Institutes of Health, Bethesda, Maryland 20892
}

Phox2b-expressing neurons of the retrotrapezoid nucleus (RTN), located in the ventrolateral brainstem, are sensitive to changes in $\mathrm{PCO}_{2} / \mathrm{pH}$, have excitatory projections to the central respiratory rhythm/pattern generator, and their activation enhances central respiratory drive. Using in vivo (conscious and anesthetized rats) and in situ (arterially perfused rat brainstem-spinal cord preparations) models, we evaluated the functional significance of this neuronal population for both resting respiratory activity and the $\mathrm{CO}_{2}$-evoked respiratory responses by reversibly inhibiting these neurons using the insect peptide allatostatin following transduction with a lentiviral construct to express the G-protein-coupled Drosophila allatostatin receptor. Selective inhibition of the Phox $2 \mathrm{~b}$-expressing neurons in the ventrolateral brainstem, including the RTN, using allatostatin was without effect on resting respiratory activity in conscious rats, but decreased the amplitude of the phrenic nerve discharge in anesthetized rats and the in situ rat preparations. Postinspiratory activity was also reduced in situ. In the absence or presence of the peripheral chemoreceptor input, inhibiting the Phox $2 \mathrm{~b}$-expressing neurons during hypercapnia abolished the $\mathrm{CO}_{2}$-evoked abdominal expiratory activity in anesthetized rats and in situ preparations. Inspiratory responses evoked by rising levels of $\mathrm{CO}_{2}$ in the breathing air were also reduced in anesthetized rats with denervated carotid bodies and conscious rats with peripheral chemoreceptors intact (by $28 \%$ and $60 \%$, respectively). These data indicate a crucial dependence of central expiratory drive upon Phox $2 \mathrm{~b}$-expressing neurons of the ventrolateral brainstem and support the hypothesis that these neurons contribute in a significant manner to $\mathrm{CO}_{2}$-evoked increases of inspiratory activity.

\section{Introduction}

The retrotrapezoid nucleus (RTN) constitutes a part of the brainstem ventral respiratory column and overlaps anatomically with a putative respiratory oscillator network identified in neonatal rat in vitro brainstem-spinal cord preparations as the parafacial respiratory group (pFRG) (Onimaru and Homma, 2003). The RTN contains a population of $\mathrm{pH}$-responsive neurons that either reside within the marginal layer of the ventral medullary surface (VMS) or have extensive projections to it (Mulkey et al., 2004). These neurons express the transcription factor Phox $2 \mathrm{~b}$, have excitatory projections to the respiratory rhythm/pattern generator, and, when activated, evoke increases in respiratory activity (Abbott et al., 2009). In humans, Phox $2 \mathrm{~b}$ mutations are associated

\footnotetext{
Received June 18, 2010; revised July 20, 2010; accepted Aug. 1, 2010.

This study was supported by The Wellcome Trust and the National Institute of Neurological Disorders and Stroke (NINDS). E.E.N. and A.L. are supported by National Heart, Lung, and Blood Institute Grant HL 28066. S.T. is supported by the Medical Research Council (Grant G0600928). J.F.R.P. is the recipient of a Royal Society Wolfson Research Merit Award, and A.V.G. is a Wellcome Trust Senior Research Fellow (Grant 079040).

*N.M. and A.P.A. contributed equally to this work.

Correspondence should be addressed to either Julian F. R. Paton or Alexander V. Gourine at the above addresses, E-mail: Julian.F.R.Paton@Bristol.ac.uk or a.gourine@ucl.ac.uk.

DOI:10.1523/JNEUROSCI.3141-10.2010

Copyright $\odot 2010$ the authors $\quad 0270-6474 / 10 / 3012466-08 \$ 15.00 / 0$
}

with the congenital central hypoventilation syndrome, which is characterized by a deficiency in central respiratory chemoreception and a broad array of autonomic abnormalities (Brunet and Pattyn, 2002; Amiel et al., 2003). Mice with targeted mutations in Phox $2 \mathrm{~b}$ display an irregular breathing pattern, do not respond to $\mathrm{CO}_{2}$, and die shortly after birth (Dubreuil et al., 2008).

This evidence suggests that RTN Phox2b-expressing neurons may play an important role in the respiratory responses induced by changes in blood and brain levels of $\mathrm{PCO}_{2} /\left[\mathrm{H}^{+}\right]$. However, the hypothesis that these neurons play a key role as central respiratory $\mathrm{CO}_{2}$ chemoreceptors (Guyenet, 2008) still lacks a key piece of evidence, namely whether acute, selective inhibition of RTN Phox2bexpressing neurons abolishes $\mathrm{CO}_{2}$-induced increases in ventilation.

Here, we targeted Phox 2b-expressing neurons in the ventrolateral region of the brainstem, which encompasses the RTN, with a lentiviral construct to express G-protein-coupled Drosophila allatostatin receptor (AlstR) (Birgül et al., 1999) under the control of the PRSx8 promoter (Abbott et al., 2009). Activation of AlstR produces a strong sustained neuronal hyperpolarization via opening of inwardly rectifying $\mathrm{K}^{+}$channels (Lechner et al., 2002; Callaway, 2005). Naturally, AlstRs are only expressed in insects and allatostatin itself does not appear to interfere with 
endogenous receptor complexes in mammals (Birgül et al., 1999; Lechner et al., 2002). In anesthetized and artificially ventilated adult rats with denervated peripheral chemoreceptors, in situ arterially perfused brainstem-spinal cord preparations of juvenile rats, and in conscious adult rats, we used allatostatin to acutely inhibit AlstR-expressing Phox $2 \mathrm{~b}$ neurons in the ventrolateral brainstem regions, including the RTN, and we studied the effects of this treatment on both resting breathing and $\mathrm{CO}_{2}$-evoked activation of inspiratory and expiratory drives.

\section{Materials and Methods}

Experiments were performed according to the UK Home Office (Scientific Procedures) Act (1986).

Viral vectors. The lentiviral vector (LVV) system used was HIV-1derived and pseudotyped with the VSV-G envelope (Coleman et al., 2003). The plasmid pTYF-PRSx8-AlstR-IRES2-EGFP was cloned into the LVV. PRSx8 is an artificial promoter for driving expression in Phox $2 \mathrm{~b}$ neurons (Teschemacher et al., 2008; Abbott et al., 2009). Titers of PRSx8-AlstREGFP-LV and the control vector (PRSx8-EGFP-LV) were between $1 \times 10^{9}$ and $1 \times 10^{10}$ transducing units $\mathrm{ml}^{-1}$. Viral concentration and titration were performed as described in detail previously (Coleman et al., 2003).

In vivo gene transfer. Weaned male Sprague Dawley or Wistar rats $(n=$ 42) weighing 50-55 g were anesthetized with a mixture of ketamine (60 $\mathrm{mg}$ of $\mathrm{kg}^{-1}$; i.m.) and medetomidine $\left(250 \mu \mathrm{g} \mathrm{kg}^{-1}\right.$, i.m. $)$. Animals were placed in a stereotaxic frame (tooth bar $-18 \mathrm{~mm}$ below the interaural line) and the RTN area received two microinjections per side $(0.25 \mu \mathrm{l}$ of each, $0.1 \mu \mathrm{l} \mathrm{min}{ }^{-1}$ ) of PRSx8-AlstR-EGFP-LV, PRSx8-EGFP-LV or a mixture of PRSx8-AlstR-EGFP-LV and PRSx8-EGFP-LV (titer ratio $4: 1$ ). The injection pipette was angled at $24^{\circ}$ and $16^{\circ}$ and injections were made -3.7 and $-3.5 \mathrm{~mm}$ ventral from calamus scriptorius, respectively, and $\pm 1.7 \mathrm{~mm}$ lateral from the midline. Anesthesia was reversed with atipamezole $\left(1 \mathrm{mg} \mathrm{kg}^{-1}\right)$. No complications were observed after the surgery and the animals gained weight normally.

Experiments in anesthetized rats. Three to 5 weeks after the injections, the animals were anesthetized with urethane $\left(1.15 \mathrm{~g} \mathrm{~kg}^{-1}\right.$; i.v.) following femoral vein cannulation under isoflurane $(3 \%)$ induction. Core temperature was kept at $37.0 \pm 0.2^{\circ} \mathrm{C}$. The trachea was cannulated and the animal was ventilated artificially $(1 \mathrm{~Hz}, 1.5-2 \mathrm{ml})$ with $\mathrm{O}_{2}$-enriched air $\left(\sim 30 \% \mathrm{O}_{2}\right)$. End-tidal level of $\mathrm{CO}_{2}$ was monitored continuously (Capstar-100; CWE) and blood gases measured regularly. The ventral brainstem surface was exposed as described previously (Gourine et al., 2005a,b) and the carotid sinus and aortic nerves were sectioned to eliminate inputs from the peripheral chemoreceptors. Phrenic nerve activity (PNA) and abdominal electromyogram $\left(\mathrm{EMG}_{\mathrm{ABD}}\right)$ were recorded as indicators of central inspiratory and expiratory drives, respectively. Signals were amplified $(\times 20,000$ and $\times 5000$, respectively), filtered $(800-1000$ and $10-800 \mathrm{~Hz}$, respectively), rectified, and smoothed. Artificial CSF (aCSF; saturated with $95 \% \mathrm{O}_{2} / 5 \% \mathrm{CO}_{2}, \mathrm{pH} 7.4$ ) or allatostatin $(10 \mu \mathrm{M}$ in aCSF; Ser-Arg-Pro-Tyr-Ser-Phe-Gly-Leu- $\mathrm{NH}_{2}$; Phoenix Pharmaceuticals) was applied to the ventral brainstem surface of rats transduced with either PRSx8-AlstR-EGFP-LV or PRSx8-EGFP-LV and the effect of this treatment on PNA and $\mathrm{EMG}_{\mathrm{ABD}}$ was determined during normocapnia and at the peak of the $\mathrm{CO}_{2}$-evoked response. In a separate experimental para$\operatorname{digm}(n=6)$, the $\mathrm{CO}_{2}$-evoked response was first evaluated after application of aCSF on the brainstem surface, followed by a subsequent $\mathrm{CO}_{2}$ challenge in the presence of allatostatin $(10 \mu \mathrm{M})$, and then another hypercapnic test after allatostatin washout from the brainstem surface with aCSF.

In situ perfused brainstem-spinal cord preparations. Experiments using in situ perfused brainstem-spinal cord preparations (Paton, 1996; Trapp et al., 2008; Abdala et al., 2009) were conducted 2-3 weeks after the injections of the virus. In brief, the rats $(n=10)$ were given heparin (1000 U, i.p.), anesthetized deeply with halothane until loss of paw withdrawal reflex, bisected under the diaphragm, immersed in cold carbogenated Ringer solution, and decerebrated precollicularly. Preparations were then transferred to a recording chamber and a double lumen cannula was placed into the descending aorta for retrograde perfusion with a Ringer solution containing the following (in mM): $125 \mathrm{NaCl}, 24 \mathrm{NaHCO}_{3}, 3 \mathrm{KCl}$, $2.5 \mathrm{CaCl}_{2}, 1.25 \mathrm{MgSO}_{4}, 1.25 \mathrm{KH}_{2} \mathrm{PO}_{4}$, and 10 D-glucose. Ficoll (MWt
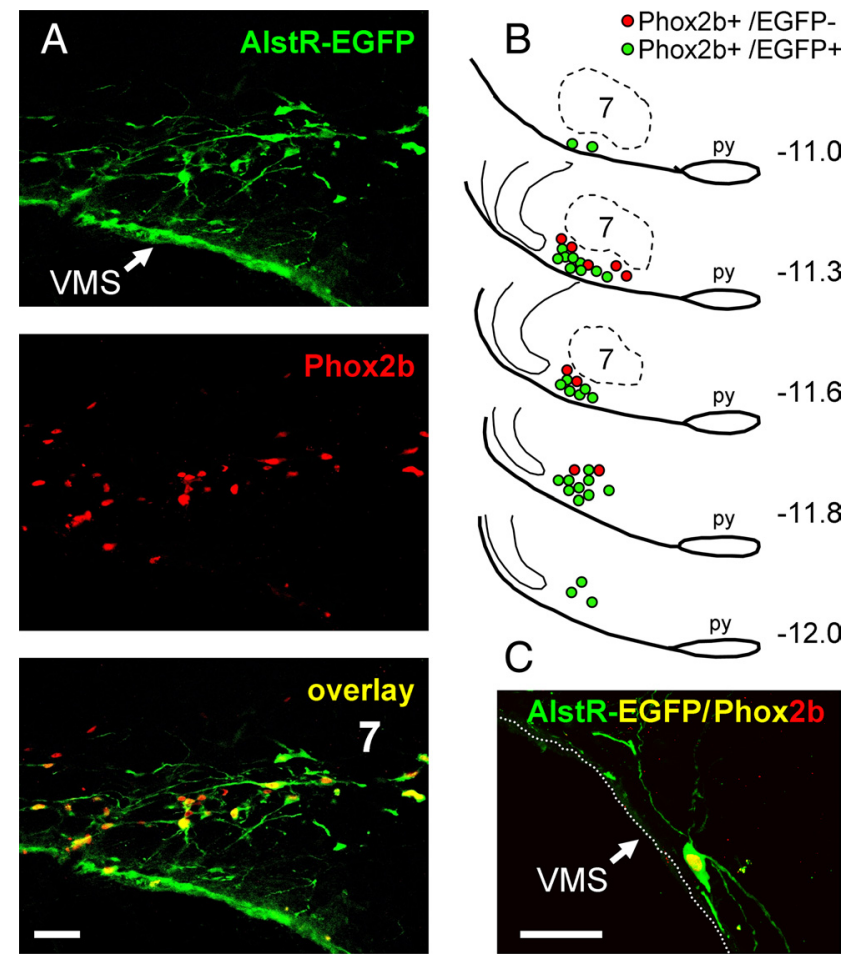

Figure 1. Targeting Phox2b-expressing ventrolateral brainstem neurons using PRSx8AlstR-EGFP-LV. A, Confocal images of Phox2b-immunoreactive nuclei (red) expressing AlstREGFP (green). Colocalization appears as yellow. Bregma level, $-11.3 \mathrm{~mm}$. $\boldsymbol{B}$, Distribution of Phox $2 \mathrm{~b}$ neurons expressing AlstR-EGFP transgene in one representative rat 5 weeks after microinjection of PRSx8-AlstR-EGFP-LV into the ventrolateral brainstem. Each symbol represents three neurons. Numbers on the right indicate distances from bregma. $C$, Phox2b-immunoreactive neuron expressing AlstR-EGFP residing within the VMS marginal layer. 7, Facial motor nucleus; py, pyramidal tract. Scale bars, $50 \mu \mathrm{m}$.

20,$000 ; 1.25 \%$ ) was added as an oncotic agent and the perfusion solution was saturated with $95 \% \mathrm{O}_{2} / 5 \% \mathrm{CO}_{2}$ (pH 7.35-7.4; osmolality $290 \pm 5$ mosm $\times \mathrm{kg} \mathrm{H}_{2} \mathrm{O}^{-1}$ at $31^{\circ} \mathrm{C}$ ). Carotid body input was intact. PN, central vagus $(\mathrm{cVN})$, hypoglossal $(\mathrm{HN})$, and abdominal (AbN; T13/L1 level) nerve activities were simultaneously recorded. Signals were amplified $(\times 20,000)$, filtered $(60-3000 \mathrm{~Hz})$, rectified, and integrated $(50 \mathrm{~ms}$ time constant). To inhibit transduced Phox $2 \mathrm{~b}$-expressing neurons, allatostatin (1 $\mu \mathrm{M})$ was added to the perfusate. Fresh perfusate was used to washout the peptide. Eight or $10 \% \mathrm{CO}_{2}$ (in $92 \%$ or $90 \% \mathrm{O}_{2}$, respectively) was used as the hypercapnic stimuli. Throughout the experiments, perfusion pressure was maintained constant by adjusting the perfusion flow rate.

Experiments in conscious rats. Animals transduced into the ventrolateral brainstem with either PRSx8-AlstR-EGFP-LV or PRSx8-EGFP-LV were reanesthetized (3-5 weeks later) with the mixture of ketamine and medetomidine. An indwelling cannula (Plastics One) was implanted into the lateral cerebral ventricle; a subcutaneous EMG electrode was sutured into the diaphragm $\left(\mathrm{EMG}_{\mathrm{DIA}}\right)$ and the peripheral lead/connector was tunneled under the skin and exited behind the head. Animals were allowed to recover for 7-10 $\mathrm{d}$ before being habituated to a recording chamber. Allatostatin (2 $\mathrm{mM}, 10 \mu \mathrm{l}$ ) was administered intracerebroventricullarly (i.c.v.) and $\mathrm{EMG}_{\text {DIA }}$ responses were recorded under normal conditions (air breathing) and during hypercapnia (inspired gas mixture containing $6 \% \mathrm{CO}_{2}$, $21 \% \mathrm{O}_{2}, 73 \% \mathrm{~N}_{2}$ ).

Immunohistochemistry. At the end of the experiments, animals were perfused transcardially with $0.9 \% \mathrm{NaCl}$ solution followed by $500 \mathrm{ml}$ of cold $4 \%$ paraformaldehyde (PFA). Brainstems were postfixed overnight in $4 \%$ PFA at $4^{\circ} \mathrm{C}$ and cryoprotected in $30 \%$ sucrose. A one in three series of $30 \mu \mathrm{m}$ coronal sections was used for immunohistochemical detection of Phox $2 \mathrm{~b}$ and EGFP. Tissue was incubated in chicken anti-GFP antibody (1:250; Avés Labs) for $48 \mathrm{~h}$ followed by rabbit anti-Phox $2 \mathrm{~b}$ antiserum for 24 h (1:800; a generous gift from J. F. Brunet, Ecole Normale 
Supérieure, Paris, France). Secondary antibodies were goat anti-chicken Alexa 568 (1:1000; Molecular Probes) and biotinylated goat antirabbit (1:500) amplified by fluorescein avidin DCS (1:250; Vector Laboratories). For presentation purposes, images were captured on a LCS510 Zeiss confocal microscope and pseudocolored in green (EGFP) and red (Phox $2 \mathrm{~b})$.

Recordings of RTN neurons in vitro and in situ. EGFP fluorescence in cells transduced with PRSx8-AlstR-EGFP-LV had been found to be rather weak. Therefore, for these experiments, the rats were injected with a mixture of viral vectors PRSx8-AlstR-EGFP-LV and PRSx8-EGFP-LV. Cotransduction with both vectors drove the expression of AlstR and bright fluorescent EGFP in the same cells, allowing visually guided patch-clamp recordings from the targeted neuronal population. A ratio of 4:1 transducing units $\mathrm{ml}^{-1}$ of PRSx8-AlstREGFP-LV to PRSx8-EGFP-LV was used to ensure that identified fluorescent cells were expressing AlstR. One to 2 weeks after the injections, rats were killed with halothane overdose and $250 \mu \mathrm{m}$ coronal brainstem slices were cut and maintained in vitro, as described previously (Hopwood and Trapp, 2005). Patch pipettes (3-6 $\mathrm{M} \Omega$ ) were pulled from thin-walled borosilicate capillary glass (Clark Electromedical Instruments). Electrodes were filled with the following (in $\mathrm{mM}$ ): $120 \mathrm{~K}$-gluconate, 5 HEPES, 5 BAPTA, $1 \mathrm{NaCl}, 1 \mathrm{MgCl}_{2}, 1 \mathrm{CaCl}_{2}, 2$ $\mathrm{K}_{2} \mathrm{ATP}$. Recordings were performed under an epifluorescence microscope (Zeiss Axioskop 2 FS; Zeiss) in aCSF saturated with $95 \% \mathrm{O}_{2} / 5 \%$ $\mathrm{CO}_{2}, \mathrm{pH} 7.4$, at $28-32^{\circ} \mathrm{C}$. The recording chamber (volume, $2 \mathrm{ml}$ ) was perfused with aCSF at a rate of $4-5 \mathrm{ml} \mathrm{min}^{-1}$. Hypercapnia was induced by saturating the perfusate with $90 \% \mathrm{O}_{2} /$ $10 \% \mathrm{CO}_{2}$. Transduced neurons were identified by EGFP fluorescence and were selected by their location in relation to facial nucleus and the VMS. Recordings were performed in cell-attached configuration and whole-cell current-clamp mode using an EPC-9 amplifier and Pulse/ Pulsefit software (Heka Elektronik). Currents or membrane potentials were filtered at $1 \mathrm{kHz}$ and digitized at $3 \mathrm{kHz}$.

The effects of allatostatin on the activity of RTN neurons were also tested in in situ perfused brainstem-spinal cord preparations of rats transduced with PRSx8-AlstR-EGFP-LV 10 d prior. $\mathrm{CO}_{2}$-sensitive neurons were identified and recorded in the RTN area using a custom-made, three-barrel, glass electrode ( $15 \mathrm{M} \Omega$ ). To aid identification of the chemosensitive RTN neurons, the perfusion solution was saturated with a gas mixture containing elevated $\mathrm{CO}_{2}\left(94 \% \mathrm{O}_{2} / 6 \% \mathrm{CO}_{2}\right)$. The recording barrel was filled with $3 \mathrm{M} \mathrm{NaCl}$ and the other two barrels were filled with glutamate ( $10 \mathrm{~mm}$ in aCSF, to verify sufficient proximity to neuron) and allatostatin (1 mM in aCSF). Both barrels were connected to a pneumatic drug ejection system (PDES-2L; NPI Electronics) and the drugs were picoejected using pressurized nitrogen $(100 \mathrm{kPa})$ as described previously (Pierrefiche et al., 2007).

Analysis. Physiological data were digitized ( $3 \mathrm{kHz}$ sampling rate) and analyzed offline (Spike2; CED). The effects of allatostatin on the activities of the phrenic nerve (anesthetized and in situ), $\mathrm{EMG}_{\text {DIA }}$ (conscious), $\mathrm{AbN}$ (EMG or nerve in anesthetized and in situ preparations, respectively), $\mathrm{HN}$ (in situ), and cVN (duration of postinspiration; in situ) under resting conditions and during hypercapnia were analyzed using Student's paired $t$ test. Repeated-measures ANOVA was applied to the sequential data obtained in conscious rats. The area under the curve for integrated $\mathrm{cVN}$ and $\mathrm{AbN}$ postinspiratory activities in situ was calculated. Mean normalized values are presented. A $p$ value of $<0.05$ was considered significant. allatostatin

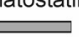
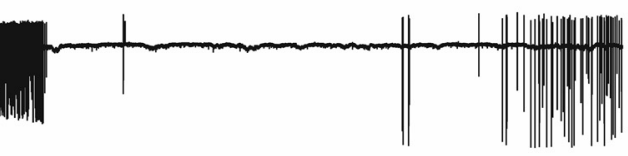

tostatin

$1 \mathrm{~min}$

C
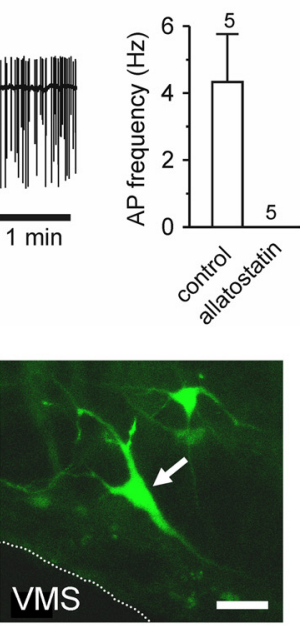

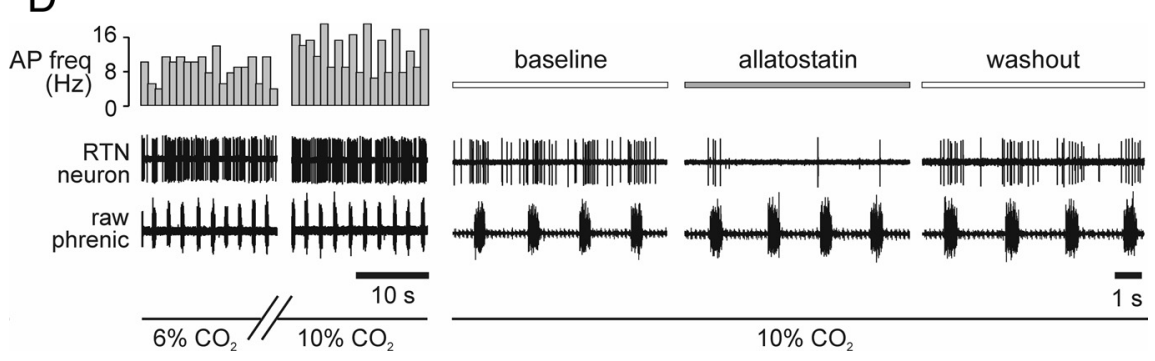

Figure 2. Acute silencing of individual ventrolateral brainstem Phox2b neurons expressing AlstR-EGFP by allatostatin. $\boldsymbol{A}$, Representative cell-attached recording from an EGFP-positive ventrolateral brainstem neuron (left). As the mean data (right)

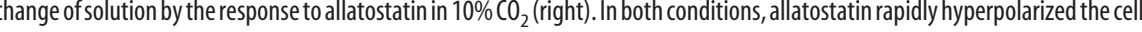
extracellular recording of $\mathrm{CO}_{2}$-sensitive RTN neuron in the in situ brainstem-spinal cord preparation. Chemosensitivity of the neuron is shown by the increased firing frequency at $10 \% \mathrm{CO}_{2}$. Allatostatin application produced a significant decrease in spiking activity of RTN neurons at high $\mathrm{CO}_{2}(n=4)$. AP freq, Action-potential frequency.

Anatomical distribution of the EGFP-immunoreactive (IR) neurons and Phox2b-IR nuclei was analyzed with epifluorescence microscopy. Colocalization of Phox2b-IR nuclei with EGFP immunostaining was determined by visual inspection through a $20 \times$ objective. Cell counts were performed manually using one in three series of $30 \mu \mathrm{m}$ brainstem sections (an average of 14 brainstem slices per rat was used). Phox2b-IR cells transduced with PRSx8-AlstR-EGFP-LV were counted on both sides regardless of staining intensity. Cell numbers were summed and pooled in two groups according to their position relative to bregma and using the caudal border of the facial nucleus as anatomical reference. Efficacy of the transduction is expressed as a percentage of Phox 2b-IR neurons expressing AlstREGFP located rostrally and caudally from the caudal border of the facial nucleus. Anatomical reconstruction of EGFP-expressing Phox $2 b$ neurons in a representative brainstem was performed manually using the Paxinos and Watson stereotaxic atlas as a guide.

\section{Results}

Targeting Phox $2 b$-expressing neurons in the ventrolateral brainstem using PRSx8-AlstR-EGFP-LV

Transduction efficacy was examined histologically in the brainstems of all the rats used for the experiments performed with anesthetized in vivo preparations $(n=14)$ and the in situ perfused preparations $(n=10)$. No differences in the regional transduction pattern indicated by AlstR-EGFP-IR and Phox $2 \mathrm{~b}-\mathrm{IR}$ expression were found, consistent with the fact that the same-size animals, same stereotaxic apparatus, and same viral injection co- 

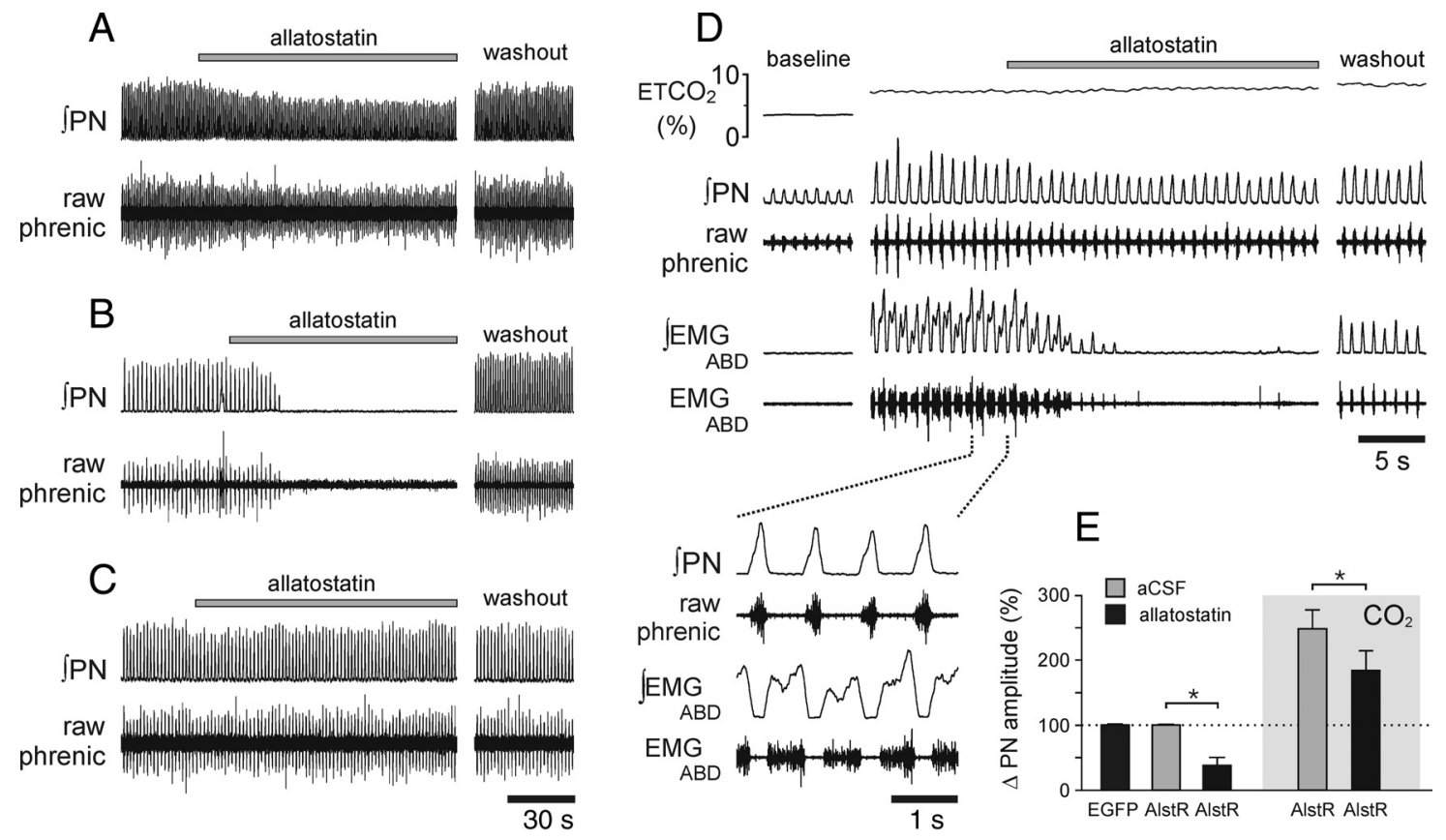

Figure 3. Acute inhibition of AlstR-expressing Phox2b neurons in the ventrolateral brainstem reduces phrenic nerve amplitude and abolishes $\mathrm{CO}_{2}$-evoked abdominal expiratory activity in anesthetized rats. $\boldsymbol{A}$, Representative raw data illustrating changes in phrenic nerve amplitude in response to allatostatin (10 $\mu \mathrm{M})$ application on the ventral surface of the brainstem in rats transduced with PRSx8-AlstR-EGFP-LV and breathing normally. B, Silencing of the phrenic nerve discharge in response to allatostatin application in an animal transduced with PRSx8-AlstR-EGFP-LV in the ventrolateral brainstem and kept just above apneic threshold by mechanical ventilation $(35 \mathrm{mmHg} \mathrm{PaCO}$; pHa, 7.45). C, Allatostatin (2 mM) application on the VMS does not produce significant changes in phrenic nerve output in animals transduced with PRSx8-EGFP-LV. D, Representative raw data illustrating changes in phrenic nerve amplitude and abdominal EMG in response to allatostatin applied on the ventral brainstem surface at the peak of the $\mathrm{CO}_{2}$-evoked response in an animal transduced with PRSx8-AlstR-EGFP-LV. E, Summary data showing changes in phrenic nerve amplitude induced by allatostatin application on the ventral brainstem surface in animals transduced with PRSx8-AlstR-EGFP-LV (AlstR) or PRSx8-EGFP-LV (EGFP) during normocapnia and hypercapnia. ${ }^{*} p<0.05$.

ordinates were used in all of these rats. EGFP immunostaining revealed significant expression of AlstR-EGFP in the targeted ventrolateral brainstem region that encompasses the RTN. EGFP immunofluorescence was located both in the soma and processes of the Phox2b-IR neurons (Fig. 1A,C). Many transduced cells identified in the anatomical region of the RTN displayed morphological features of chemosensitive neurons (Mulkey et al., 2004), with cell bodies located in close proximity to the VMS and dendritic tree projecting toward the marginal layer (Fig. 1C). A representative distribution of the transduced neurons in a sequence of brainstem sections is shown in Figure $1 B$. Transduced neurons were distributed in an area of $\sim 2 \mathrm{~mm}$ along the rostrocaudal extent of the rostral ventrolateral medulla. A large number of transduced neurons was concentrated at the level of the facial nucleus in the area corresponding to RTN and located at $\sim 11.3$ mm caudal to bregma (Kang et al., 2007). An average of $4.5 \%$ of EGFP-IR neurons did not show a clearly defined Phox $2 b-I R$ nucleus. We suggest, however, that these neurons are in fact false Phox $2 \mathrm{~b}$-negative. Indeed, some of the cells have relatively large elongated bodies and may have been divided during sectioning, resulting in slices that contain substantial parts of the cellular body and no Phox 2b-positive nucleus (which remained in the next or previous slice in the sequence).

We detected an average of $229 \pm 61$ Phox2b-positive AlstREGFP-expressing neurons per side after counting Phox 2b-IR nuclei in every third section ( $n=24$ reconstructed brainstems). This translates into an estimate of $\sim 1400$ Phox 2 b-expressing neurons per brainstem. Rostral clusters of EGFP-IR neurons were distributed in close proximity to the VMS in a ventral to ventrolateral position relative to the facial nucleus (Fig. 1B). Analysis of these sections revealed that $64 \pm 10 \%$ of identified
Phox2b-expressing neurons located rostral to the caudal border of the facial nucleus were transduced. The percentage of transduced Phox2b-IR neurons was slightly lower $(50 \pm 8 \%)$ in ventrolateral medullary areas located caudal to the facial nucleus, in the area corresponding to the location of catecholaminergic $\mathrm{C} 1$ cell group. In accord with the data reported by Abbott et al. (2009) using similar LVV constructs, these results confirm significant expression of AlstR-EGFP in the ventrolateral brainstem Phox2b-expressing neurons, including the RTN and $\mathrm{C} 1$ cellular populations.

\section{Silencing AlstR-EGFP-transduced Phox2b-expressing ventrolateral brainstem neurons by allatostatin}

EGFP-expressing neurons located near the VMS were selected for patch-clamp recordings (Fig. 2C). The effects of allatostatin (1 $\mu \mathrm{M}$ ) on spontaneous electrical activity were tested using extracellular recordings in the cell-attached patch-clamp configuration $(n=5)$ and under whole-cell current-clamp conditions $(n=5)$. RTN neurons in cell attached configuration had a mean firing rate of $4.3 \pm 1.4 \mathrm{~Hz}$ under resting conditions $\left(5 \% \mathrm{CO}_{2}\right)$. Application of allatostatin $(1 \mu \mathrm{M})$ reversibly abolished actionpotential firing within $1 \mathrm{~min}$ in every cell tested (Fig. $2 A$ ). In whole-cell current-clamp recording configuration, cells fired spontaneous action potentials at a frequency of $2.7 \pm 1.3 \mathrm{~Hz}(n=$ 5). Allatostatin induced a $-8.0 \pm 2.1 \mathrm{mV}$ hyperpolarization and abolished action-potential generation in all of the recorded neurons (Fig. $2 \mathrm{~B}$ ). After allatostatin washout and recovery for $\sim 30$ min, two of these neurons remained sufficiently stable and were exposed to aCSF saturated with $90 \% \mathrm{O}_{2} / 10 \% \mathrm{CO}_{2}$, which augmented basal firing rate (Fig. $2 \mathrm{~B}$ ). Subsequent application of allatostatin in high- $\mathrm{CO}_{2}$ conditions led to hyperpolarization (by -5 and $-9 \mathrm{mV}$ ) and cessation of action-potential firing in both 
tested neurons (Fig. 2 B). Allatostatin had no effect on five EGFPnegative RTN neurons under the same experimental conditions (data not shown). In an in situ brainstem spinal cord preparation, activities of the $\mathrm{CO}_{2}$-responsive neurons identified and recorded from within the anatomical region of the RTN were significantly and reversibly inhibited by allatostatin $(n=4)$ (Fig. $2 D)$.

Inhibition of Phox $2 \mathrm{~b}$-expressing neurons in the ventrolateral brainstem decreases resting respiratory activity and $\mathrm{CO}_{2}$-evoked increases in phrenic nerve discharge

In anesthetized, carotid body denervated, and artificially ventilated rats expressing AlstR-EGFP in the ventrolateral brainstem $(n=8)$, application of allatostatin $(10 \mu \mathrm{M})$ onto the ventral brainstem surface during normal breathing resulted in a reduction in phrenic nerve discharge amplitude (by $57 \% ; p<0.01$ ) (Fig. $3 A, E$ ). Allatostatin had no effect on the respiratory rate in animals with intact vagi because respiratory frequency was entrained to the ventilator. In animals transduced with PRSx8AlstR-EGFP-LV, which were kept just above the apneic threshold by facilitated mechanical ventilation arterial $\mathrm{pH}(\mathrm{pHa}) 7.45$ (35 $\mathrm{mmHg} \mathrm{PaCO}_{2}$ ), application of allatostatin produced a complete silencing of phrenic nerve discharge (Fig. 3B). Allatostatin (in concentrations of up to $2 \mathrm{mM}$ ) had no effect on the respiratory activity in animals transduced with PRSx8-EGFP-LV (in vivo, $n=5$; in situ, $n=3$ ) (Fig. 3C) or in animals injected with PRSx8-AlstREGFP-LV $1 \mathrm{~mm}$ rostral to the facial nucleus and showing no expression of the transgene in the RTN area (in vivo, $n=5$; in situ, $n=4$ ) (data not shown). Therefore, allatostatin does not appear to crossreact with endogenous receptors within the brainstem respiratory circuits, confirming previously reported data (Tan et al., 2008).

In conditions of increased central respiratory drive, e.g., at the peak of the $\mathrm{CO}_{2}$-evoked response, allatostatin application onto the brainstem surface of rats expressing AlstR-EGFP in the ventrolateral brainstem reduced the amplitude of the phrenic nerve discharge by $28 \%(p<0.01)$ (Fig. $3 D, E)$. In a separate protocol, allatostatin was applied to the brainstem surface of animals $(n=$ 6) transduced with PRSx8-AlstR-EGFP-LV into the ventrolateral brainstem before the $\mathrm{CO}_{2}$ stimulus was applied. In the presence of allatostatin, an increase in the level of inspired $\mathrm{CO}_{2}$ still evoked vigorous increases in phrenic nerve discharge (Fig. 4), which were, however, significantly smaller $(-9 \%)$ compared with control $\mathrm{CO}_{2}$ challenges $(p<0.05)$.

In the in situ preparations $(n=10)$, administration of allatostatin under normocapnia reduced phrenic nerve discharge amplitude (by $38 \% ; p<0.01$ ) (Fig. $5 A, C$ ) but increased its frequency (by 96\%; $p<0.01$ ) (Fig. $5 A$ ); therefore, neural minute inspiration (product of integrated $\mathrm{PN}$ discharge frequency and amplitude) was not affected. Integrated $\mathrm{HN}$ amplitude decreased (by $44 \% ; p<0.001$ ), as did postinspiratory activity of the cVN nerve (by $84 \% ; p<0.0001$ ) (Fig. $5 A, C)$. Hypercapnia $\left(8 \% \mathrm{CO}_{2}\right)$ increased phrenic nerve amplitude (by 25\%; $p<0.01$ ) (Fig. $5 C$ ), frequency (by 29\%; $p<0.01$ ), and neural minute ventilation (by $61 \%, p<0.001$ ) (Fig. $5 B$ ), but high $\mathrm{CO}_{2}$ in the presence of allatostatin decreased phrenic nerve amplitude (by $-7 \% ; p<0.05$ ) and increased inspiratory frequency by $90 \%(p<0.01)$ and, hence, neural minute inspiration was significantly elevated (by $75 \%, p<0.01$ ) (Fig. 5). Thus, in the in situ preparation, the increase in neural minute inspiration induced by hypercapnia was not affected by inhibition of Phox $2 b$-expressing ventrolateral brainstem neurons.

In conscious rats transduced into the ventrolateral brainstem with PRSx8-AlstR-EGFP-LV $(n=6)$, the resting respiratory fre-

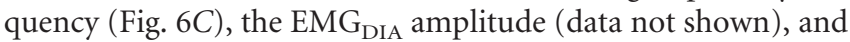

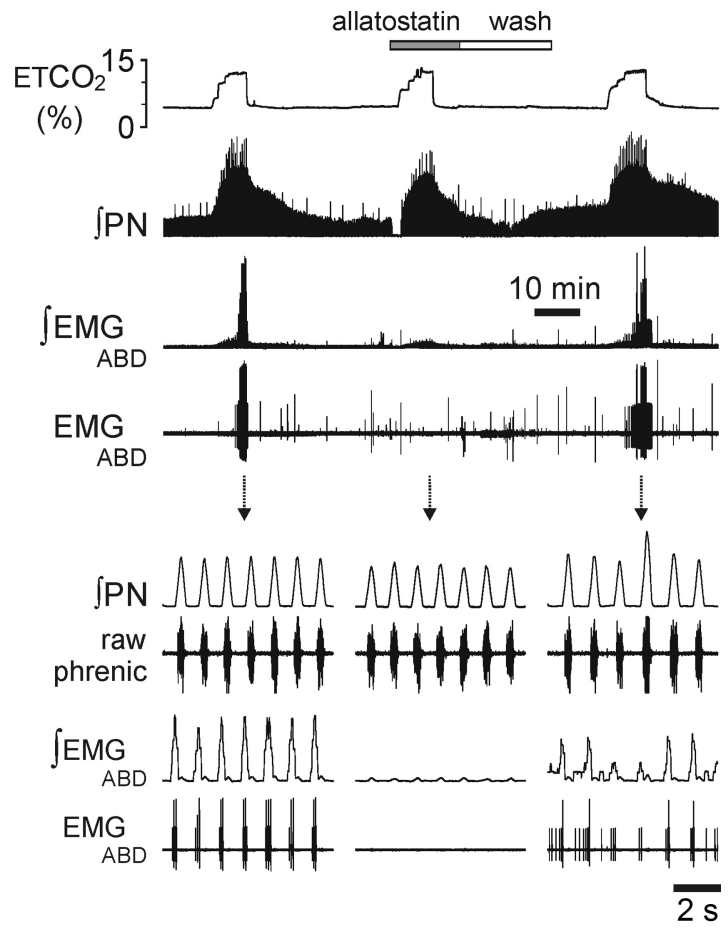

Figure 4. Hypercapnia fails to trigger expiratory activity following acute inhibition of AlstRexpressing Phox2b neurons in the ventrolateral brainstem in anesthetized rats. Timecondensed record (top traces) illustrating changes in integrated PN amplitude and abdominal EMG in response to repeated increases in the level of inspired $\mathrm{CO}_{2}$ in the absence and presence of allatostatin $(10 \mu \mathrm{M})$ on the ventral brainstem surface in an animal transduced with PRSx8AlstR-EGFP-LV. Expanded time base recordings of PN and abdominal EMG are shown below, illustrating late-expiratory abdominal activity evoked by hypercapnia and reversibly blocked by allatostatin application to the ventral brainstem.

minute respiration (the product of frequency and $\mathrm{EMG}_{\mathrm{DIA}}$ amplitude) (Fig. 6C) were unaffected following i.c.v. administration of allatostatin $(2 \mathrm{~mm} ; 10 \mu \mathrm{l})$. During hypercapnia, allatostatin pretreatment reduced the peak $\mathrm{CO}_{2}$-induced increases in respiratory rate and minute respiration $40 \mathrm{~min}$ after the injection by $19.2 \pm 4.2$ and $39.0 \pm 8.7 \%$, respectively (Fig. $6 C$ ), and attenuated the percentage increases in respiratory frequency from $59.8 \pm 8.9 \%$ to $32.7 \pm 5.7 \%(p<0.05)$ and in minute respiration from $194.7 \pm 45.1 \%$ to $77.9 \pm 11.5 \%(p<0.05)$. Thus, in conscious animals with intact peripheral chemoreceptor inputs, allatostatin actions on EGFP-AlstR transduced ventral brainstem Phox $2 \mathrm{~b}$-expressing neurons results in a substantial effect on the $\mathrm{CO}_{2}$-evoked respiratory response ( $\sim 60 \%$ reduction). Partial recovery of the $\mathrm{CO}_{2}$ response was observed $75 \mathrm{~min}$ after allatostatin injections (Fig. 6).

Inhibition of Phox 2b-expressing neurons in the ventrolateral brainstem abolishes expiratory activities evoked by $\mathrm{CO}_{2}$

Under resting, normocapnic conditions, expiratory abdominal activity is either absent (anesthetized rats in vivo) or of a small amplitude (in situ preparations) (Figs. 3D, 4, 5A). However, robust active expirations are triggered (as recorded from the abdominal muscles/nerves) by systemic hypercapnia (Figs. 3D, 4, $5 B)$. In anesthetized PRSx8-AlstR-EGFP-LV transduced rats $(n=$ 8 ), allatostatin applied onto the ventral surface of the brainstem at the peak of the $\mathrm{CO}_{2}$ response reversibly abolished $\mathrm{EMG}_{\mathrm{ABD}}$ activities (both postinspiratory and late expiratory activity) (Fig. 3D). Furthermore, $\mathrm{CO}_{2}$ failed to evoke $\mathrm{EMG}_{\mathrm{ABD}}$ activity when allatostatin was given before the application of the $\mathrm{CO}_{2}$ stimulus 
A

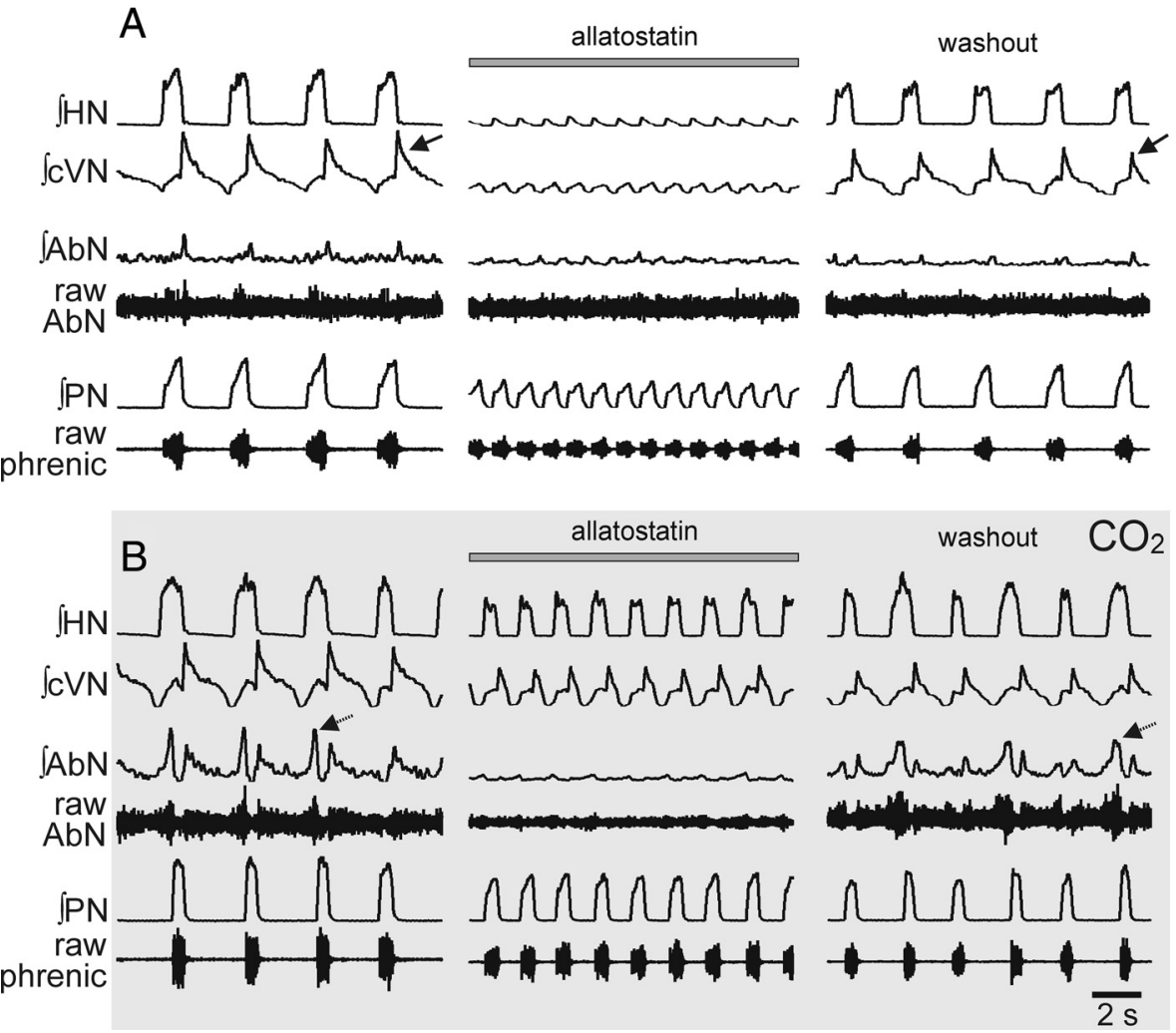

C
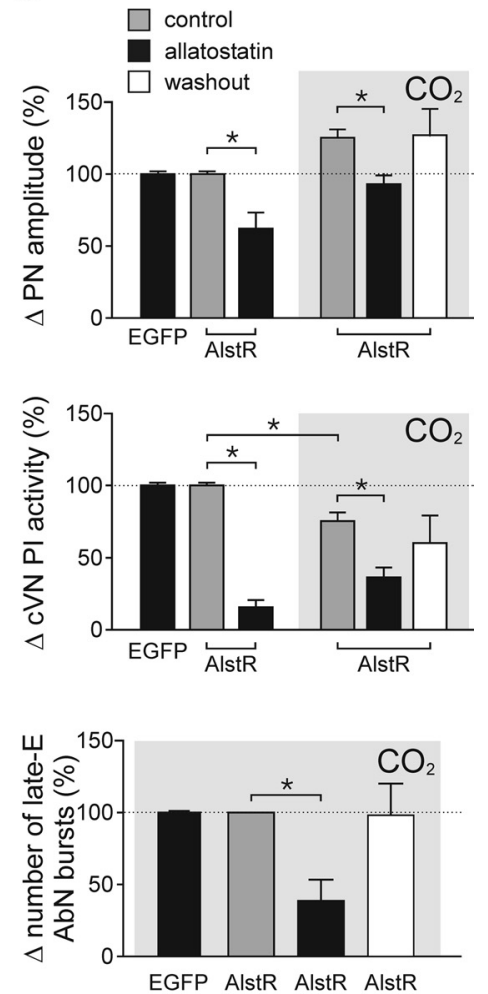

Figure 5. Acute inhibition of AlstR-expressing Phox2b neurons in the ventrolateral brainstem prevents $\mathrm{CO}_{2}$-induced postinspiratory vagal and late-expiratory abdominal discharges in situ. $A$, Changes in resting $\mathrm{HN}$, cVN, AbN, and PN activities following allatostatin (1 $\mu \mathrm{m})$ administration via the perfusate in an in situ preparation from an animal transduced with PRSX8-AlstR-EGFP-LV in the ventrolateral brainstem. Note reversible reduction of inspiratory and postinspiratory (arrow) activities; the latter accounts for the increase in respiratory frequency. $\boldsymbol{B}$, The response to $8 \% \mathrm{CO}_{2}$ in the absence and presence of allatostatin; note the almost complete abolition of AbN late expiratory activity (arrows) and partial suppression of postinspiratory cVN activity. The reduction in inspiratory amplitude was less than that seen in normocapnia (see $A)$. C, Summary data showing the effect of allatostatin on $\mathrm{CO}_{2}$-induced increases in phrenic nerve amplitude, postinspiratory cVN activity, and numbers of AbN late-expiratory (late-E) bursts in preparations from the animals transduced with PRSx8-AlstR-EGFP-LV (AlstR) or PRSx8-EGFP-LV (EGFP) in the ventrolateral brainstem. ${ }^{*} p<0.05$. Allatostatin application in non-LVV injected rats or rats transduced with PRSx8-EGFP-LV did not produce changes in respiratory motor outputs (data not shown).

$(n=6)$ (Fig. 4). Similarly, in the in situ preparations $(n=10)$, hypercapnia-evoked AbN late expiratory activity was either reduced (by $63 \%$ in amplitude and $61 \%$ in the number of bursts; $p<0.01 ; n=4$ ) (Fig. $5 C$ ) or abolished $(n=4)$ (Fig. $5 B)$ by allatostatin application. Postinspiratory activity (recorded from the $\mathrm{cVN}$ nerve) decreased in response to $\mathrm{CO}_{2}(-24 \%, p<0.01)$ and this effect was further facilitated by allatostatin treatment $(-64 \% ; p<0.001)$ (Fig. 5C).

\section{Discussion}

This report reveals an essential functional role of Phox2bexpressing neurons of the ventrolateral brainstem regions encompassing the RTN in the chemosensory control of breathing in mature animals. In the in vivo and in situ preparations in the presence or absence of peripheral chemoreceptor input, inhibiting presumably of a significant proportion of these neurons eliminated $\mathrm{CO}_{2}$-evoked expiratory activities in $\mathrm{AbN}$ and $\mathrm{cVN}$ motor outflows, and reduced, although did not abolish, $\mathrm{CO}_{2}$-evoked increases in inspiratory drive. In anesthetized rats with denervated peripheral chemoreceptors and in the in situ preparations with carotid bodies intact, inhibition of Phox $2 \mathrm{~b}$-expressing ventrolateral brainstem neurons reduced the amplitude of the phrenic nerve discharge, revealing that these neurons exert an excitatory drive to the respiratory network at rest. However, respiratory rate increased in in situ preparations (secondary to a depression of postinspiratory activity) and, hence, minute respiration was similar before and after allatostatin administration. In conscious rats, inhibition of Phox $2 \mathrm{~b}$ neurons had no effect on resting respiratory activity, although the $\mathrm{CO}_{2}$-evoked ventilatory response was significantly reduced. Together, these data indicate a crucial dependence of expiratory drive upon Phox2bexpressing neurons of the ventrolateral brainstem, including the RTN. The data also confirm that these neurons contribute to the $\mathrm{CO}_{2}$-evoked increases in the inspiratory motor output.

LVV targeting resulted in a significant level of AlstR-EGFP expression among Phox $2 \mathrm{~b}$ neurons residing in the ventrolateral brainstem regions. Histological analysis revealed that $\sim 60 \%$ of identified Phox $2 \mathrm{~b}$-expressing neurons located rostral to the caudal border of the facial nucleus (i.e., within the RTN) were transduced. It is possible, however, that we may have underestimated the actual proportion of transduced neurons, because we did not directly analyze the actual expression of AlstR. To assess transduction efficiency, we had to rely on immunohistochemical detection of EGFP. Given the magnitude of the specific physiological effect observed after application of allatostatin (i.e., complete abolishing of the late-expiratory drive), it is possible that the immunofluorescence labeling method used was not sensitive enough to detect low levels of EGFP expression in some of the transduced Phox2b-expressing neurons.

Many identified AlstR-EGFP transduced Phox2b-expressing neurons were found to be located near the VMS or within the VMS marginal layer (Fig. 1C) and displayed anatomical features of the chemosensitive RTN neurons (Mulkey et al., 2004). How- 
ever, the PRSx8 promoter is also active in other ventrolateral medullary neuronal populations, including Phox2b-expressing catecholaminergic $\mathrm{C} 1$ neurons (Lonergan et al., 2005). Although our injections were targeted to the RTN, histological reconstruction indicates that a significant proportion of more caudally located $\mathrm{C} 1$ neurons was also transduced and, therefore, inhibited after allatostatin application. Indeed, EGFPlabeled neurons were found in the ventrolateral medullary reticular formation caudal to the facial nucleus, where $\mathrm{C} 1$ neurons are distributed.

It is conceivable, therefore, that the effects of allatostatin on respiratory activities reported herein are partially due to inhibition of the transduced population of catecholaminergic $\mathrm{C} 1$ neurons. However, there is evidence that $\mathrm{C} 1$ neurons (either a subset or the whole population of them) may not be intrinsically chemosensitive-in vitro at room temperature they show no responses to changes in $\mathrm{pH}$ (Lazarenko et al., 2009). Moreover, recent work by Abbott et al. (2009) has shown that phrenic nerve responses induced by photostimulation of RTN Phox $2 b$ neurons expressing channelrhodopsin 2 under the control of the same PRSx8 promoter are identical in intact rats and in rats with $\mathrm{C} 1$ lesions. This suggests that the inspiratory responses elicited by activation of RTN neurons are independent of the activity of C1 neurons. By extension, this also suggests that even though a substantial proportion of $\mathrm{C} 1$ neurons may have been transduced by PRSx8-AlstR-EGFP-LV, allatostatin-induced inhibition of the phrenic nerve responses to $\mathrm{CO}_{2}$ is unlikely to be related to allatostatin actions on these neurons. Whether $\mathrm{C} 1$ neurons contribute to generation of the expiratory activity remains to be determined. Such a contribution is not inconceivable, since this cellular population may provide an essential drive to expiratory interneurons of the Bötzinger complex located nearby (Kanjhan et al., 1995).

Rapid inhibitory and readily reversible effects of allatostatin were previously reported to be selective for AlstR-transduced neurons (Lechner et al., 2002). In the present study, we confirm effective silencing of AlstR-expressing neurons by allatostatin in normocapnic conditions and during hypercapnia in vitro. There is a possibility that these strong effects of allatostatin in vitro are due to the nature of the preparation because, in theory, cells isolated from excitatory synaptic inputs are more easily hyperpolarized and inhibited than neurons with intact connections. However, we also demonstrate here a significant inhibition of RTN $\mathrm{CO}_{2}-$ sensitive neurons by allatostatin during hypercapnia in the in situ preparation (although we were not able to visualize/target transduced neurons directly in this preparation and the phenotype of the recorded cells was not characterized). Furthermore, in our in vivo experiments using anesthetized animals, RTN neurons are also isolated to a large extent from descending inputs from higher (non-brainstem) CNS centers (due to anesthesia) and from the major peripheral chemosensory inputs (by carotid bodies denervation). Effective inhibition of the targeted neuronal population by allatostatin in vivo is also indirectly supported by the data showing that its application to the PRSx8-AlstR-EGFP-LV transduced brainstem results in a complete silencing of $\mathrm{CO}_{2}$-evoked abdominal expiratory activity. This effect is remarkably similar to the effect of complete physical removal of the RTN by either surgical transection (Janczewski and Feldman, 2006) or by regional pharmacological inhibition (Abdala et al., 2009). Thus, the physiological data on perturbations of respiratory pattern reported herein, together with the cellular-level electrophysiological evidence provided, suggest that allatostatin application effectively inhibits a significant proportion of transduced Phox $2 \mathrm{~b}$-expressing ventrolateral brainstem neurons in vivo.

By extension, these data reveal the relative significance of Phox 2 b-expressing ventrolateral brainstem neurons in the overall respiratory response to $\mathrm{CO}_{2}$. When $\mathrm{CO}_{2}$-evoked active expirations were abolished following inhibition of the Phox $2 b$ neurons in vivo, the inspiratory activity was only partially reduced. In the in situ preparations, postinspiratory activity was reduced and the phrenic frequency was elevated by allatostatin, suggesting transition to a two-phase rhythmic respiratory pattern as discussed previously (Smith et al., 2007). We acknowledge that the in situ preparation presents a biased dependency on post-I activity in determining respiratory cycle length due, in part, to the absence of pulmonary stretch receptor feedback, and may show somewhat exaggerated frequency responses with changes in post-I activity. Nevertheless, the reduced phrenic nerve amplitudes are consistent with those changes observed in anesthetized rats. We note that the same alteration of respiratory motor output pattern (loss of post-I activity with an increase in frequency of low amplitude PN discharge) is typically induced in the in situ 
preparation by hypocapnia (Smith et al., 2007), where reduced basal activity of RTN chemosensory neurons is expected.

The data obtained also demonstrate the existence of an excitatory drive from the Phox $2 \mathrm{~b}$-expressing ventrolateral brainstem neurons to the inspiratory rhythm/pattern generator. This is supported by the evidence that allatostatin produced central apnea in AlstR-EGFPtransduced anesthetized rats with denervated peripheral chemoreceptors kept just above apneic threshold. Similarly, experiments using a nonspecific toxin (Takakura et al., 2008) demonstrated a correlation between the elevation of apneic threshold and the percentage of RTN neurons permanently eliminated. In contrast to these observations, we found that inhibition of Phox $2 \mathrm{~b}$-expressing ventrolateral brainstem neurons, including those of the RTN, in conscious rats had no effect on baseline respiratory activity, but significantly decreased the respiratory response to an elevated level of inspired $\mathrm{CO}_{2}$. In general, these data are in agreement with the results of previous studies, some of which report decreases in resting ventilation but all of which report decreased $\mathrm{CO}_{2}$-evoked respiratory responses following pharmacological or neurotoxic inhibition of RTN neurons/circuits, albeit by approaches nonspecific to Phox2bexpressing neurons (Nattie and Li, 2009).

Highly pH-chemosensitive neurons, identified in the RTN in vivo (Mulkey et al., 2004), are tonically active over a wide range of $\mathrm{PaCO}_{2}$, showing weak respiratory modulation of their discharge. Thus, these neurons are unlikely to generate the $\mathrm{CO}_{2}$-evoked expiratory rhythm. However, when $\mathrm{PaCO}_{2}$ increases, these neurons are fully equipped to provide powerful tonic excitatory input to the expiratory oscillator/controller located nearby within the pFRG (Onimaru and Homma, 2003; Janczewski and Feldman, 2006; Abdala et al., 2009), Bötzinger complex, (Abdala et al., 2009) or directly to the caudal ventral respiratory group of the medulla for onward relay to expiratory spinal motoneurons.

In summary, our data reveal a distinct physiological function of a population of Phox $2 b$-expressing neurons of the ventrolateral brainstem, including $\mathrm{pH}$-sensitive RTN neurons. They control recruitment of abdominal expiratory muscles during forced breathing, such as during systemic hypercapnia, and contribute, in part, to $\mathrm{CO}_{2}$-evoked increases of inspiratory motor activity. We propose that a separate component of the $\mathrm{CO}_{2}$ drive for inspiration is likely to arrive from the chemosensitive elements located in other parts of the brainstem (Nattie, 1999; Richerson, 2004; Nattie and $\mathrm{Li}, 2009$ ) and/or RTN neurons that do not express Phox $2 \mathrm{~b}$. These results point to a fundamental integrative function of the Phox2b-expressing neurons of the ventrolateral brainstem in the generation and coordination of inspiratory and expiratory activities associated with the chemosensory control of breathing.

\section{References}

Abbott SB, Stornetta RL, Fortuna MG, Depuy SD, West GH, Harris TE, Guyenet PG (2009) Photostimulation of retrotrapezoid nucleus Phox2b-expressing neurons in vivo produces long-lasting activation of breathing in rats. J Neurosci 29:5806-5819.

Abdala AP, Rybak IA, Smith JC, Paton JF (2009) Abdominal expiratory activity in the rat brainstem-spinal cord in situ: patterns, origins and implications for respiratory rhythm generation. J Physiol 587:3539-3559.

Amiel J, Laudier B, Attié-Bitach T, Trang H, de Pontual L, Gener B, Trochet D, Etchevers H, Ray P, Simonneau M, Vekemans M, Munnich A, Gaultier C, Lyonnet S (2003) Polyalanine expansion and frameshift mutations of the paired-like homeobox gene PHOX2B in congenital central hypoventilation syndrome. Nat Genet 33:459-461.

Birgül N, Weise C, Kreienkamp HJ, Richter D (1999) Reverse physiology in Drosophila: identification of a novel allatostatin-like neuropeptide and its cognate receptor structurally related to the mammalian somatostatin/ galanin/opioid receptor family. EMBO J 18:5892-5900.
Brunet JF, Pattyn A (2002) Phox2 genes: from patterning to connectivity. Curr Opin Genet Dev 12:435-440.

Callaway EM (2005) A molecular and genetic arsenal for systems neuroscience. Trends Neurosci 28:196-201.

Coleman JE, Huentelman MJ, Kasparov S, Metcalfe BL, Paton JF, Katovich MJ, Semple-Rowland SL, Raizada MK (2003) Efficient large-scale production and concentration of HIV-1-based lentiviral vectors for use in vivo. Physiol Genomics 12:221-228.

Dubreuil V, Ramanantsoa N, Trochet D, Vaubourg V, Amiel J, Gallego J, Brunet JF, Goridis C (2008) A human mutation in Phox $2 b$ causes lack of $\mathrm{CO}_{2}$ chemosensitivity, fatal central apnea, and specific loss of parafacial neurons. Proc Natl Acad Sci U S A 105:1067-1072.

Gourine AV, Llaudet E, Dale N, Spyer KM (2005a) ATP is a mediator of chemosensory transduction in the central nervous system. Nature 436:108-111.

Gourine AV, Llaudet E, Dale N, Spyer KM (2005b) Release of ATP in the ventral medulla during hypoxia in rats: role in hypoxic ventilatory response. J Neurosci 25:1211-1218.

Guyenet PG (2008) The 2008 Carl Ludwig Lecture: retrotrapezoid nucleus, $\mathrm{CO}_{2}$ homeostasis, and breathing automaticity. J Appl Physiol 105:404-416.

Hopwood SE, Trapp S (2005) TASK-like K+ channels mediate effects of $5-\mathrm{HT}$ and extracellular $\mathrm{pH}$ in rat dorsal vagal neurones in vitro. J Physiol 568:145-154.

Janczewski WA, Feldman JL (2006) Distinct rhythm generators for inspiration and expiration in the juvenile rat. J Physiol 570:407-420.

Kang BJ, Chang DA, Mackay DD, West GH, Moreira TS, Takakura AC, Gwilt JM, Guyenet PG, Stornetta RL (2007) Central nervous system distribution of the transcription factor Phox $2 \mathrm{~b}$ in the adult rat. J Comp Neurol 503:627-641.

Kanjhan R, Lipski J, Kruszewska B, Rong W (1995) A comparative study of pre-sympathetic and Botzinger neurons in the rostral ventrolateral medulla (RVLM) of the rat. Brain Res 699:19-32.

Lazarenko RM, Milner TA, Depuy SD, Stornetta RL, West GH, Kievits JA, Bayliss DA, Guyenet PG (2009) Acid sensitivity and ultrastructure of the retrotrapezoid nucleus in Phox2b-EGFP transgenic mice. J Comp Neurol 517:69-86.

Lechner HA, Lein ES, Callaway EM (2002) A genetic method for selective and quickly reversible silencing of mammalian neurons. J Neurosci 22:5287-5290.

Lonergan T, Teschemacher AG, Hwang DY, Kim KS, Pickering AE, Kasparov S (2005) Targeting brainstem centres of cardiovascular control using adenoviral vectors: impact of promoters on transgene expression. Physiol Genomics 20:165-172.

Mulkey DK, Stornetta RL, Weston MC, Simmons JR, Parker A, Bayliss DA, Guyenet PG (2004) Respiratory control by ventral surface chemoreceptor neurons in rats. Nat Neurosci 7:1360-1369.

Nattie E (1999) $\mathrm{CO}_{2}$, brainstem chemoreceptors and breathing. Prog Neurobiol 59:299-331.

Nattie E, Li A (2009) Central chemoreception is a complex system function that involves multiple brain stem sites. J Appl Physiol 106:1464-1466.

Onimaru H, Homma I (2003) A novel functional neuron group for respiratory rhythm generation in the ventral medulla. J Neurosci 23:1478-1486.

Paton JF (1996) A working heart-brainstem preparation of the mouse. J Neurosci Methods 65:63-68.

Pierrefiche O, Abdala AP, Paton JF (2007) Nitric oxide and respiratory rhythm in mammals: a new modulator of phase transition? Biochem Soc Trans 35:1258-1263.

Richerson GB (2004) Serotonergic neurons as carbon dioxide sensors that maintain pH homeostasis. Nat Rev Neurosci 5:449-461.

Smith JC, Abdala AP, Koizumi H, Rybak IA, Paton JF (2007) Spatial and functional architecture of the mammalian brain stem respiratory network: a hierarchy of three oscillatory mechanisms. J Neurophysiol 98:3370-3387.

Takakura AC, Moreira TS, Stornetta RL, West GH, Gwilt JM, Guyenet PG (2008) Selective lesion of retrotrapezoid Phox2b-expressing neurons raises the apnoeic threshold in rats. J Physiol 586:2975-2991.

Tan W, Janczewski WA, Yang P, Shao XM, Callaway EM, Feldman JL (2008) Silencing preBotzinger complex somatostatin-expressing neurons induces persistent apnea in awake rat. Nat Neurosci 11:538-540.

Teschemacher AG, Wang S, Raizada MK, Paton JF, Kasparov S (2008) Areaspecific differences in transmitter release in central catecholaminergic neurons of spontaneously hypertensive rats. Hypertension 52:351-358.

Trapp S, Aller MI, Wisden W, Gourine AV (2008) A role for TASK-1 (KCNK3) channels in the chemosensory control of breathing. J Neurosci 28:8844-8850. 